\title{
Successful pregnancy without disease progression of radioiodine refractory papillary thyroid carcinoma: a case report
}

\author{
Yuchen Jin, Min Liu, Lingxiao Cheng and Libo Chen * (1)
}

\begin{abstract}
Background: Pregnancy is an unquantifiable risk to accelerate tumor growth of papillary thyroid carcinoma (PTC), and whether pregnancy induces an unfavorable prognosis of radioiodine refractory papillary thyroid carcinoma (RR-PTC) remains unknown.

Case presentation: We investigated the impact of pregnancy on the prognosis of pulmonary metastases in an RR-PTC woman via a long-term clinical follow-up and consecutive computed tomography examinations and serum tests. After a successful pregnancy, the metastatic lesions shrank with serum thyroglobulin slightly fluctuated under sustained thyroid stimulating hormone (TSH) suppression, demonstrating a favorable outcome.

Conclusions: This case study indicates that metastatic RR-PTC may not be aggravated by pregnancy under TSH suppression, and pregnancy should not be contraindicated in RR-PTC patients with stable disease.

Keywords: Radioiodine refractory papillary thyroid carcinoma, Pregnancy, Prognosis, Thyroid stimulating hormone, Thyroglobulin
\end{abstract}

\section{Background}

With the development of diagnostic technology, increasing number of patients was diagnosed as radioiodine refractory papillary thyroid carcinoma (RR-PTC) with relatively poor prognosis [1]. However, to date, few data can be referred to predict the outcome of RR-PTC in patients who will undergo pregnancies. To bring a conclusion, an analysis of work-flow from our database registering for radioiodine $\left({ }^{131} \mathrm{I}\right)$ treatment (Jan. 2014Dec. 2016, $n=876$ ) has been made. After excluding males $(n=269)$, pathological types other than PTC $(80)$, patients with no pregnancy history $(n=224)$, patients with pregnancy before ${ }^{131}$ I treatment $(n=276)$, loss of follow-up $(n=14)$, pregnant patients without evidence of metastasis $(n=9)$, miscarriage before ${ }^{131}$ I remnant ablation $(n=3)$, there was only one patient finally included. Herein, we describe the RR-PTC case with pulmonary metastases who underwent a complete

\footnotetext{
* Correspondence: libochen888@hotmail.com

Department of Nuclear Medicine, Shanghai Jiao Tong University Affiliated Sixth People's Hospital, Yishan Rd. 600, Shanghai 200233, People's Republic of China
}

(c) The Author(s). 2017 Open Access This article is distributed under the terms of the Creative Commons Attribution 4.0 International License (http://creativecommons.org/licenses/by/4.0/), which permits unrestricted use, distribution, and reproduction in any medium, provided you give appropriate credit to the original author(s) and the source, provide a link to the Creative Commons license, and indicate if changes were made. The Creative Commons Public Domain Dedication waiver (http://creativecommons.org/publicdomain/zero/1.0/) applies to the data made available in this article, unless otherwise stated. pregnancy and documents its impact on the prognosis of the disease.

\section{Case presentation}

A 26-year-old female who complained of cervical nodules was referred to our hospital in Nov. 2012. PTC was then verified by ultrasound-guided fine needle aspiration cytology and multiple pulmonary nodules were found by thoracic computed tomography $(\mathrm{CT})$. The patient then received near-total thyroidectomy and lymph node dissection. In the year 2013, consecutive administrations of ${ }^{131}$ I were given in Jan. and Jun. for remnant ablation (3700 MBq) and treatment of pulmonary metastasis (7400 MBq). Post-ablation ${ }^{131}$ I whole body scan (WBS) showed only thyroid remnant uptake (Fig. 1a) and posttherapy WBS (Fig. 1b) revealed no ${ }^{131} \mathrm{I}$-avid foci. Before the second administration of ${ }^{131} \mathrm{I}$, the thyroglobulin $(\mathrm{Tg})$ level under thyroid stimulating hormone (TSH) stimulation peaked to $1493 \mathrm{ng} / \mathrm{mL}$ with normal anti-Tg antibody level and stable findings of chest CT.

About 7 months after the last administration of ${ }^{131} \mathrm{I}$, she got pregnant. In Sep. 2014, cesarean section was performed at 36 weeks' gestation because of oligohydramnios. 

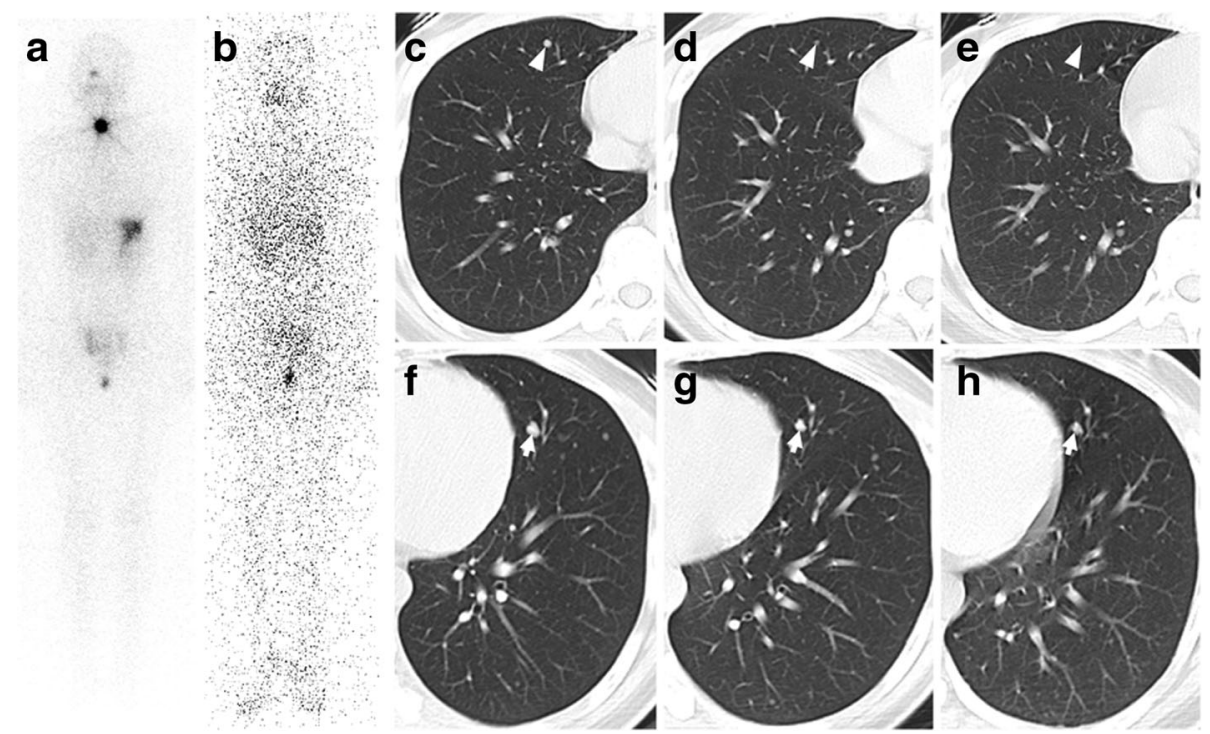

Fig. 1 Imaging examinations. Whole body scintigraphy 3 days post the two doses of ${ }^{131}$ I (a 100 mCi for remnant ablation; b $200 \mathrm{mCi}$ for cancer therapy) showed that there were no ${ }^{131}$ I-avid foci in the bilateral lungs (b). Diagnostic CT imaging (c and $\mathbf{f}, 3$ months before getting pregnant, the maximum diameters of the largest lesions in the right and left lung were $3.75 \mathrm{~mm}$ and $6.66 \mathrm{~mm}$, respectively; $\mathbf{d}$ and $\mathbf{g}, 21$ months post cesarean section, the maximum diameters of the largest lesions in the right and left lung were $1.83 \mathrm{~mm}$ and $5.34 \mathrm{~mm}$, respectively; e and $\mathbf{h}$, 30 months post cesarean section, the largest lesion in the right lung was not seen and the maximum diameter of the largest lesion in the left lung was $4.88 \mathrm{~mm}$ ) revealed shrinkage of metastatic foci without new lesions. Arrowhead, the largest lesion in the right lung; Arrow, the largest lesion in the left lung

The patient delivered a healthy male infant $(3 \mathrm{~kg})$ with Apgar score of 10 and normal TSH level. Compared with pregestational data (baseline, Oct. 2013) (Fig. 1c and f, Fig. 2), Tg value at 10 months after cesarean section (Jul. 2015) fluctuated slightly (+8\%) from 65.76 to $70.99 \mathrm{ng} / \mathrm{L}$ (Fig. 2) with evident shrinkage of pulmonary foci without new lesions demonstrated by CT (Fig. 1d and g). Thirty months (Mar. 2017) after cesarean section, serum tests revealed TSH of $0.02 \mathrm{mIU} / \mathrm{L}, \mathrm{Tg}$ of $86.38 \mathrm{ng} / \mathrm{mL}$ and $\mathrm{TgAb}$ of $12.14 \mathrm{IU} / \mathrm{mL}$, and $\mathrm{CT}$ examination indicated further improvement of the disease (Fig. 1e and h).

Additionally, the patient felt well before, during and after gestation at continuous TSH suppression status (0.01-0.71 $\mathrm{mIU} / \mathrm{L})$ sustained by oral administration of levothyroxine. At the time of this writing, the 32-monthold child was healthy.

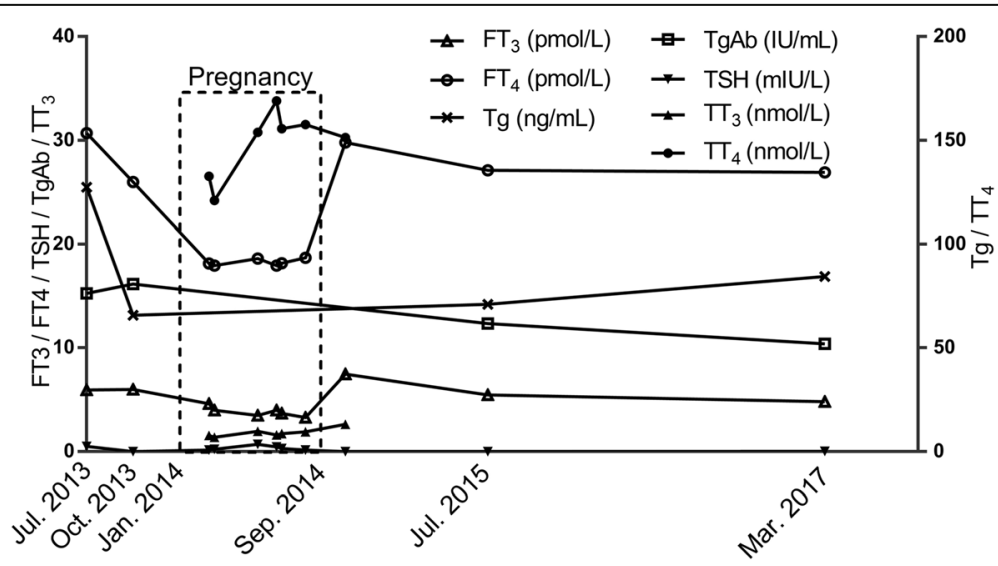

Fig. 2 Serum tests. Serum thyroglobulin fluctuated insignificantly with sustained suppressed TSH before, during and after pregnancy. Square frame represents the period of pregnancy (Jan. 2014-Sep. 2014). $\mathrm{FT}_{3}$, free triiodothyronine (normal: 3.67-6.00 pmol/L); $\mathrm{FT}_{4}$, free thyroxine (normal: 7.50-21.10 pmol/L); TSH, thyroid stimulating hormone (normal: 0.34-5.60 mlU/L); Tg, thyroglobulin (normal: 3.50-77.00 ng/mL); TgAb, anti-Tg antibody (normal: $0.00-115.00 \mathrm{IU} / \mathrm{mL}$ ); $\Pi_{3}$, total triiodothyronine (normal: $1.13-2.42 \mathrm{nmol} / \mathrm{L}$ ); $\Pi_{4}$, total thyroxine (normal: $75.37-167.78 \mathrm{nmol} / \mathrm{L}$ ) 


\section{Discussion and conclusions}

Pregnancy is generally an important unquantifiable risk to maternal health, which has the potential for accelerating tumor growth of PTC due to proliferative effects of fluctuating TSH, estrogen (E2) and human chorionic gonadotropin (hCG) as reported previously [2]. During pregnancy, although the fluctuation of hormones is complicated, the net effect on the prognosis of well differentiated PTC may be favorable. Some scholars believe that pregnancy does not appear to induce a poor prognosis of PTC. Most clinical outcome data also showed no difference in the rate of recurrence or long-term survival of women with well-differentiated PTC identified during pregnancy [3-5]. Sturniolo $G$ et al. observed an association between ER- $\alpha$ expression and a more favorable outcome in PTC patients [6]. In addition, Rowe et al. described a favorable outcome in a pregnant woman with metastatic PTC, who gave a normal birth of a healthy male child weighing $2380 \mathrm{~g}$ at 34 weeks of gestation [7]. Although two doubling rises of Tg was observed in a 33year-old woman with pT2pN1bMx PTC during her consecutive trimesters, Tg levels returned to her prepregnancy baseline level following each delivery [8].

Although the prognosis of RR-PTC is poorer than well differentiated individuals, patients may also live for a long time with stable disease [1]. Therefore, the impact of potential pregnancy on the prognosis of RR-PTC should be disclosed. To the best of our knowledge, this is the first RR-PTC patient with pulmonary metastases who went through a successful pregnancy without disease progression, which was assessed by both biomarker and structural modality. As is described above, clinical follow-up in combination with consecutive thoracic CT scans and laboratory analyses revealed an outcome of stable disease. Interestingly, pulmonary metastases shrank after gestation, indicating that pregnancy per se may also be a favorable factor for the prognosis of RRDTC patients.

In summary, this case study indicates that metastatic RR-PTC may not be aggravated by pregnancy under TSH suppression, and pregnancy should not be contraindicated in RR-PTC patients with stable disease. Longer-term follow-up and more sufficient investigations are still needed.

\section{Abbreviations}

CT: computed tomography; E2: estrogen; hCG: human chorionic gonadotropin; PTC: papillary thyroid carcinoma; RR-PTC: radioiodine refractory papillary thyroid carcinoma; TSH: thyroid stimulating hormone

\section{Acknowledgments}

We thank Prof. Weijie Sun from Department of Obstetrics and Gynecology, Peking University First Hospital and Dr. Lianhuan Zhang from Department of Endocrinology, Shaoxing Hospital of Traditional Chinese Medicine for helpful comments and data collection.

\section{Funding}

This study was sponsored by the National Natural Science Foundation of China (81671711 and 81271609) and the Shanghai Rising-Star Program $(12 \mathrm{QH} 1401600)$ in the data collection and analysis, as well as in writing the manuscript.

\section{Availability of data and materials}

The data analyzed are available from the corresponding author on reasonable request.

\section{Authors' contributions}

YCJ wrote the manuscript and all authors carefully revised the manuscript. $M L$ and LXC cared for and followed up the patient. LBC managed this case and gave his expert recommendations. All authors have read and approved the final version of this manuscript.

\section{Authors' information}

Department of Nuclear Medicine, Shanghai Jiao Tong University Affiliated Sixth People's Hospital Yishan Rd. 600, Shanghai 200,233, People's Republic of China.

\section{Ethics approval and consent to participate}

The need for ethics approval was waived by the ethics committee of Shanghai Jiao Tong University Affiliated Sixth People's Hospital due to the retrospective nature of the study.

\section{Consent for publication}

Informed consent was written by the patient for publication of the accompanying data, and a copy is available for review.

\section{Competing interests}

The authors declare that they have no competing of interests.

\section{Publisher's Note}

Springer Nature remains neutral with regard to jurisdictional claims in published maps and institutional affiliations.

Received: 11 June 2017 Accepted: 30 October 2017

Published online: 09 November 2017

\section{References}

1. Capdevila J, Galofre JC, Grande E, Zafon Llopis C, Ramon YCAT, Navarro Gonzalez E, Jimenez-Fonseca P, Santamaria Sandi J, Gomez Saez JM, Riesco Eizaguirre $\mathrm{G}$. Consensus on the management of advanced radioactive iodinerefractory differentiated thyroid cancer on behalf of the Spanish Society of Endocrinology Thyroid Cancer Working Group (GTSEEN) and Spanish rare cancer working group (GETHI). Clin Transl Oncol. 2017;19(3):279-87.

2. Khaled H, Al Lahloubi N, Rashad N. A review on thyroid cancer during pregnancy: multitasking is required. J Adv Res. 2016;7(4):565-70.

3. Haugen BR, Alexander EK, Bible KC, Doherty GM, Mandel SJ, Nikiforov YE, Pacini F, Randolph GW, Sawka AM, Schlumberger M, et al. 2015 American Thyroid Association management guidelines for adult patients with thyroid nodules and differentiated thyroid cancer: the American Thyroid Association guidelines task force on thyroid nodules and differentiated thyroid cancer. Thyroid : official journal of the American Thyroid Association. 2016;26(1):1-133.

4. Moosa M, Mazzaferri EL. Outcome of differentiated thyroid cancer diagnosed in pregnant women. J Clin Endocrinol Metab. 1997;82(9):2862-6.

5. Yasmeen S, Cress R, Romano PS, Xing G, Berger-Chen S, Danielsen B, Smith LH. Thyroid cancer in pregnancy. International journal of gynaecology and obstetrics: the official organ of the International Federation of Gynaecology and Obstetrics. 2005;91(1):15-20.

6. Sturniolo G, Zafon C, Moleti M, Castellvi J, Vermiglio F, Mesa J. Immunohistochemical expression of estrogen receptor-alpha and progesterone receptor in patients with papillary thyroid cancer. European thyroid journal. 2016;5(4):224-30.

7. Rowe CW, Murray K, woods a, Gupta S, smith R, Wynne K. management of metastatic thyroid cancer in pregnancy: risk and uncertainty. Endocrinol Diabetes Metab Case Rep. 2016:2016

8. Murray JR, Williams GR, Harrington KJ, Newbold K, Nutting CM. Rising thyroglobulin tumour marker during pregnancy in a thyroid cancer patient: no cause for alarm? Clin Endocrinol. 2012;77(1):155-7. 